\title{
Artículos
}

\section{Manejo del estrés y miedo al COVID-19 desde la integración de los enfoques cognitivo-conductual y centrado}

\author{
en soluciones
}

\section{Management of stress and fear of Covid-19 from the integration of cognitive-behavioral and solution-focused}

\author{
therapies
}

\begin{abstract}
Blanca María Gómez Hoyt; Karla Patricia Méndez Pruneda; Miriam Fernanda Novak Villarreal; Mónica Teresa González Ramírez
\end{abstract}

Universidad Autónoma de Nuevo León

\section{Resumen}

El presente estudio evalúa un taller en línea basado en la terapia que integra el enfoque cognitivo-conductual y el centrado en soluciones para manejar el estrés y el miedo de contagio de COVID-19. Participaron 10 personas (1 hombre y 9 mujeres) de entre 19 y 60 años con un total de 5 sesiones semanales. Los resultados de las evaluaciones (escala de miedo al COVID-19, cuestionario de impacto psicológico del coronavirus y escala de estrés percibido relacionado con la pandemia de COVID-19) mostraron una disminución del estrés percibido y del miedo hacia el COVID-19. El tamaño del efecto encontrado al comparar los puntajes iniciales y finales respalda la conclusión de que el taller en línea puede considerarse una buena opción para tratar temas sobre estrés y miedo.

Palabras clave: COVID-19, miedo, estrés, intervención.

Blanca María Gómez Hoyt. Facultad de Psicología, Universidad Autónoma de Nuevo León

Karla Patricia Méndez Pruneda. Facultad de Psicología, Universidad Autónoma de Nuevo León Miriam Fernanda Novak Villarreal. Facultad de Psicología, Universidad Autónoma de Nuevo León Mónica Teresa González Ramírez. Facultad de Psicología, Universidad Autónoma de Nuevo León https://orcid.org/0000-0002-9058-9626

Autor para correspondencia:

Mónica Teresa González Ramírez

Correo electrónico: monygzz77@yahoo.com; monica.gonzalezrz@uanl.edu.mx 


\begin{abstract}
The present study evaluates an online workshop, using an integrative therapy approach combining cognitive behavioral and solution focused therapy, to manage stress and fear of contagion of COVID-19. Ten people ( 1 man and 9 women) with an age range between 19 and 60 years old participated in a total of 5 weekly sessions. Results of the assessments (Fear of COVID-19 Scale, Coronavirus Psychological Impact Questionnaire and Pandemic-Related Perceived Stress Scale of COVID-19) showed a decrease in perceived stress and fear of COVID-19. The effect size found when comparing the initial and final scores supports the conclusion that the online workshop can be considered a good option to address issues of stress and fear.

Keywords: COVID-19, fear, stress, intervention.
\end{abstract}

DOI https://doi.org/10.36793/psicumex.v11i2.408

Recibido 8 - Enero - 2021

Aceptado 16 - Junio - 2021

Publicado 25 - Septiembre -2021 


\section{Introducción}

El SARS-COV-2 (COVID-19) es una neumonía epidémica originada en Wuhan, China a finales de diciembre de 2019. En enero de 2020, la Organización Mundial de la Salud declaró esto como una emergencia de salud pública de importancia internacional. Entre los síntomas clínicos más comunes de esta enfermedad están la fiebre, tos, fatiga, dificultad para respirar, dolor de garganta y dolor de cabeza (Guo et al., 2020). Lo alarmante de esta situación se debe a la rapidez del contagio, lo que provoca la saturación en centros de salud pública (Urzúa et al., 2020).

A raíz de la llegada del virus a México, el gobierno empezó a tomar medidas sanitarias como el distanciamiento social y confinamiento, provocando altos niveles de ansiedad, enojo, confusión y estrés dentro de la población (Gordon, 2020). Aunado a la incertidumbre sobre el paro en la economía y a la preocupación sobre la salud y bienestar de los que los rodean, así como la de uno mismo, la exposición constante a la información brindada por los medios de comunicación puede llegar a provocar mayores niveles de estrés, depresión, insomnio, ira y somatizaciones (Salari et al., 2020).

Estas reacciones ante la pandemia han sido objeto de estudio, Brooks et al. (2020) informan que los estresores reportados por la cuarentena son su duración, miedo a la infección, frustración, aburrimiento, falta de suministros, información inadecuada, problemas financieros y estigma. Castellini et al. (2021) encontraron un incremento en síntomas de ansiedad y depresión durante la primera etapa del confinamiento en comparación con los datos antes de la pandemia; Turna et al. (2021) encontraron que el sexo femenino, la edad (los más jóvenes) y los tratamientos previos en salud mental eran predictores de altos niveles de ansiedad, depresión y estrés en los primeros meses de la pandemia. Estos datos coinciden con lo reportado por Castellano-Tejedor et al. (2021), quienes también encontraron en las primeras etapas de la pandemia que las mujeres jóvenes preocupadas por el futuro eran quienes experimentaban los niveles más altos de estrés. El estrés se encuentra presente y puede aparecer con mayor intensidad en un tipo de población que en otra, 
por ejemplo, en aquellos que tienen un mayor riesgo de contagio de COVID-19, como el personal de la salud y los trabajadores de primera línea. Aumentando el temor, la preocupación, cambios en los patrones de sueño, entre otros (Valero Cedeño et al., 2020).

Además, las preocupaciones, miedos y/o ansiedad de las personas confinadas podrían asociarse a otros factores secundarios, como la salud de las personas queridas, el posible colapso del sistema de salud, problemas laborales y pérdidas de ingresos, la expansión mundial del virus y sus consecuencias económicas y sociales (Sandín et al., 2020).

La exposición continua al estrés en la pandemia COVID-19 afecta las funciones cognitivas, afectivas y conductuales de la persona, y si no es resuelta puede generar alteraciones metabólicas e inmunológicas que poco a poco debilitaran al organismo para hacer frente a procesos infecciosos, aumentando el riesgo de posibles enfermedades (Muñoz-Fernández et al., 2020).

El presente estudio se enmarca en la teoría transaccional del estrés, en la cual el estrés se define como una relación entre el individuo y su entorno; cuando el entorno es evaluado como amenazante, desbordante de los recursos de la persona o que pone en peligro su bienestar se desencadenan las respuestas de estrés (Lazarus y Folkman, 1984). Lazarus (2000) considera que el estrés es interdependiente con las emociones y agrega que como mínimo hay 15 variedades diferentes de emociones, entre las que se encuentran: ansiedad, temor y tristeza. Congruente con Lazarus, Caballo et al. (2006) mencionan que como respuestas emocionales al estrés se encuentran la ansiedad, el miedo, la ira y la tristeza.

La incertidumbre y miedo que se vive ante la situación del COVID-19 mantienen a las personas en constante búsqueda del control, imaginando posibles escenarios y evaluando los recursos con los que se cuenta. El miedo es la respuesta emocional que sucede cuando las personas están siendo expuestas a una situación peligrosa y real. El cuerpo genera una activación en el sistema de defensa ante la alarma, se moviliza de manera física y cognitiva para poder actuar rápidamente (Andión, 2012). De igual forma, causa 
un malestar significativo e interfiere en las áreas de funcionamiento del individuo, afectando su calidad de vida (Sierra et al., 2003).

Las respuestas emocionales pueden tener una intensidad leve o excesiva, haciendo que las personas puedan desarrollar ciertas medidas de afrontamiento con el objetivo de poder adaptarse a un nuevo entorno. En este caso, sería a la pandemia causada por el virus COVID-19. Por otro lado, también puede generar conductas desadaptativas a nivel emocional, cognitivo o conductual que solo limitan el funcionamiento de la persona y su toma de decisiones (Muñoz-Fernández et al., 2020). Muñoz-Navarro et al. (2021) encontraron que las estrategias de regulación emocional desadaptativas moderaron positivamente la ansiedad, mientras que las estrategias adaptativas moderaron negativamente la ansiedad.

Para el manejo del estrés en intervenciones grupales, la integración de los enfoques cognitivoconductual y centrado en soluciones ha sido exitosa (López Novelo y González Ramírez, 2017; Villarroel y González Ramírez, 2015). Esta integración de enfoques se utiliza desde hace una década aproximadamente, y aunque aparentemente los enfoques son contrarios en algunos aspectos, ya que el enfoque centrado en soluciones, como su nombre lo dice, se centra en las soluciones o excepciones al problema, mientras el enfoque cognitivo conductual se basa en el problema (Ramírez Treviño y Rodríguez Alcalá, 2012). Se han documentado numerosas aplicaciones de esta integración con excelentes resultados. Para una mayor descripción de esta terapia de integración se puede recurrir a los trabajos de Juárez Carrillo y González Ramírez (2016), y Ramírez Treviño y Rodríguez Alcalá (2012).

Dentro de esta terapia de integración, en el trabajo de López Novelo y González Ramírez (2017) una de las técnicas con más impacto desde el punto de vista de los participantes fue la externalización, ya que los participantes mencionaron que lograron ver a los estresores como algo independiente a ellos, y así, sentirse abiertos a la posibilidad de tomar decisiones relacionadas a los efectos de estos estresores sobre ellos. Por su parte, Villarroel y González Ramírez (2015) resaltan el uso de la técnica de psicoeducación en 
una intervención cognitivo-conductual y centrada en soluciones con resultados satisfactorios para disminuir el estrés académico en estudiantes universitarios.

Se conoce como inoculación al estrés a una estrategia de intervención cognitivo conductual que involucra un entrenamiento en afrontar al estrés a través de la práctica de ensayos y ejercicios (Ayala, 2017). La inoculación al estrés es una estrategia congruente con la teoría transaccional del estrés y se ha aplicado como tratamiento y prevención a una amplia variedad de poblaciones, en esta estrategia se enseña a los participantes a identificar distintas señales de estrés, internas y externas, para después emplear sus repertorios de afrontamiento (Meichenbaum, 1987).

Son escasos los estudios de intervención durante la pandemia por COVID-19. Zhang et al. (2020) dieron a conocer un modelo, originado en West China Hospital, que consiste en usar las plataformas de internet para lograr una integración de médicos, psiquiatras, psicólogos y trabajadores sociales para lograr una mejor intervención. Esta intervención no es solo para el paciente afectado, sino también para sus familiares y personal médico; no solo aborda a la persona durante la pandemia, sino también después del brote. Durante el brote se intervienen dos situaciones: 1) miedo a la enfermedad (hecha por médicos y psicólogos) y 2) dificultad de adaptación (hecha por psicólogos o psiquiatras). Se resalta de este modelo que a través de las redes sociales o tecnología las personas tengan la facilidad de poder pedir ayuda o atención de una manera más eficaz. El modelo consiste en cuatro grupos formando una pirámide de ayuda, empezando por la base de la pirámide, donde están los voluntarios de la comunidad, los cuales brindan apoyo psicosocial. Subiendo al siguiente nivel está la asistencia psicológica, es decir, el grupo encargado de contestar las líneas telefónicas o chats en línea para identificar y ayudar a quienes lo necesitan. De esta llamada o chat se dirigen al equipo de rescate psicológico, los cuales están para ayudar más a profundidad en la crisis y, por último, se encuentran los expertos que se encargan de dar educación sobre la salud y todo el proceso que conlleva esta pandemia. De este modelo se retoma la formación de redes y la intervención en línea principalmente. 
Muñoz-Fernández et al. (2020) propusieron una intervención en crisis compuesta por cinco componentes básicos: primero se hace contacto emocional haciendo sentir al paciente comprendido, escuchado y apoyado, además de invitarlo a dialogar, reflejar los hechos y sentimientos. Después, sigue la focalización del problema donde se dimensiona la situación, se centra en el presente y futuro inmediato a la crisis. El tercer componente es identificar y evaluar una o más soluciones inmediatas y posteriores. El siguiente es el análisis de toma de decisiones, su objetivo es implementar soluciones inmediatas descubiertas al identificar las necesidades inmediatas; en esta etapa se puede tomar una actitud facilitadora o una actitud directiva. Por último, se encuentra el seguimiento a corto plazo, concluyendo cuando el profesional de la salud se asegura de que el paciente hizo un enlace con sus necesidades.

Es así que se requieren de más estudios de intervención. Con base en lo anterior, se analizaron los síntomas de estrés y miedo por el COVID-19 para integrar un taller que ayude a su manejo durante y después de la pandemia. Por lo que el objetivo del presente estudio fue evaluar el efecto de un taller en línea sobre el estrés percibido y el miedo al COVID-19

\section{Metodología}

El estudio corresponde a un diseño cuasi experimental con evaluación pre y post intervención de un solo grupo (Montero y León, 2005).

\section{Participantes}

Para convocar a los participantes al taller, se publicó un cartel en diferentes redes sociales personales de las terapeutas (Instagram, Facebook y WhatsApp). Este incluía un número de teléfono para que los participantes se contactaran con las terapeutas que dirigieron el taller. Los criterios de inclusión fueron: participantes mayores de edad y que se sintieran identificados con el tema "estrés y miedo en tiempos de COVID-19"; no se rechazó a nadie que quisiera participar. Se comenzó el taller con 13 participantes, sin 
embargo, solo se presentan resultados de 10 ya que, por motivos personales, algunos no pudieron asistir a todas las sesiones. De los 10 participantes, 9 son mujeres y 1 es hombre, con una edad media de 31.9 años y un rango de edad de 19 a 60 años.

\section{Instrumentos}

Los instrumentos de evaluación fueron aplicados mediante Google Forms antes de iniciar el taller y al finalizar el mismo. Los cuestionarios se seleccionaron por su pertinencia al tema, aunque, al momento del presente estudio, no se encontraron datos de los mismos en población mexicana. Se aplicaron los siguientes: Cuestionario de impacto psicológico del coronavirus (CIPC) (Sandín et al., 2020). Es un cuestionario de autoinforme creado para evaluar el impacto psicológico asociado a la pandemia por COVID-19. Incluye distintas preguntas iniciales sobre características sociodemográficas y 9 escalas independientes: (1) experiencia con el coronavirus (ECOVI); (2) uso de medios de comunicación (UMC); (3) conductas asociadas al confinamiento (CAC); (4) uso de conductas preventivas (UCP); (5) escala de miedo al coronavirus (EMC); (6) escala de distrés (ED); (7) escala de síntomas de estrés postraumático (ESEP); (8) escala de interferencia por el coronavirus (EIC); (9) escala de experiencias positivas ante las adversidades (EEPA). Los coeficientes de confiabilidad oscilaron entre $\alpha=0.72$ y $\alpha=0.93$, Para el presente estudio no se utilizaron las subescalas de medios de comunicación ni de miedo al coronavirus. Se esperaría que al término del taller disminuyeran los síntomas de estrés (distrés), el estrés postraumático, la interferencia por el coronavirus y que se incrementaran las experiencias positivas.

Además, se utilizó la escala de miedo al COVID-19 (FCV-19S) (Kwasi et al., 2020), construida con base en las escalas existentes sobre miedos, evaluaciones de expertos y entrevistas a participantes; el FCV19S es útil para proporcionar información valiosa sobre el miedo al COVID-19. Consta de 7 ítems tipo Likert, con 5 opciones de respuesta que van desde totalmente en desacuerdo, hasta totalmente de acuerdo. Al sumar las respuestas, una puntuación más alta indica mayor miedo al COVID-19. Kwasi et al. (2020) 
analizaron la validez concurrente, reportan un valor $\alpha=0.82$ para la confiabilidad. Se esperaría que al término del taller disminuyera el miedo al COVID-19.

Por último, se aplicó la escala de estrés percibido relacionada con la pandemia de COVID-19 (Campo-Arias et al., 2020). Esta escala es una versión modificada de EPP-10 adaptada para la situación actual del COVID-19. Está compuesta por 10 ítems con 5 opciones de respuesta que van de nunca a siempre. Los ítems 1, 2, 3, 6, 9 y 10 se califican de manera directa de 0 a 4 y los ítems 4, 5, 7 y 8 a la inversa. CampoArias et al. (2020) reportan un alfa de 0.86. Se esperaría que al finalizar el taller disminuyera el estrés percibido.

\section{Procedimiento}

El taller consistió en 5 sesiones semanales de una hora y media de duración cada una; se llevó a cabo en la plataforma Zoom y se enmarcó en la integración de los enfoques cognitivo-conductual y centrado en soluciones (Juárez Carrillo y González Ramírez (2016; Ramírez Treviño y Rodríguez Alcalá (2012), utilizando el entrenamiento de inoculación al estrés (Meichenbaum, 1987) e integrando técnicas mencionadas previamente como la externalización (White, 1991) y la psicoeducación. A continuación, se detalla el procedimiento de cada una de las sesiones.

Sesión 1. Diseñada para identificar cómo el estrés y el miedo se presentan y repercuten en su vida diaria. Se inició con la presentación de las terapeutas y los participantes. A continuación se utilizó la psicoeducación sobre el miedo y el estrés y sobre cómo se vive durante la crisis del COVID-19, dando un espacio para compartir experiencias personales sobre esta situación. Para complementar lo aprendido se realizó una actividad para que los participantes buscaran una situación donde el miedo/estrés no se haya presentado. Se pidió como tarea el realizar este mismo ejercicio durante la semana para compartirlo la próxima sesión. 
Sesión 2. Diseñada para reconocer la relación que existe entre pensamientos, emociones y conducta, y reestructurar la cognición. Se comenzó compartiendo las excepciones que encontraron durante la semana. Después se presentó el tema de las ideas irracionales o distorsionadas, como aparecen y cómo se pueden modificar. Para continuar, se realizó una actividad donde los participantes escribían una situación donde se hubieran sentido estresados o con miedo y cuáles fueron sus pensamientos, emociones y conducta al respecto. Se habló sobre los pensamientos y emociones negativas que experimentaron durante la semana; partiendo de estas, se trabajó con reestructuración cognitiva para modificar la percepción de las experiencias a una positiva y visualizar cómo su conducta podría cambiar. Como tarea, se pidió realizar una lista sobre las emociones o pensamientos negativos que se presentaran durante la semana.

Sesión 3. Diseñada para lograr la externalización del problema y poner en práctica la relajación progresiva. Se comenzó compartiendo las excepciones que encontraron durante la semana y como se sintieron. Posteriormente, se realizó la técnica de externalización (White, 1991) de manera individual, los participantes escribieron en una hoja el nombre que le darían a los momentos donde se presenta el estrés o miedo. Posteriormente, se hicieron preguntas para crear distancia entre ellos y lo externalizado y para visualizar el problema desde otra perspectiva, como algo ajeno a ellos. Para concluir la sesión, se realizó la técnica de relajación progresiva de Jacobson (Jacobson, 1938) y se les pidió practicar esta técnica en casa.

Sesión 4. Diseñada para aprender cómo llevar a cabo una solución de problemas eficaz. Se comenzó la sesión compartiendo las excepciones que encontraron durante la semana y como se sintieron. A continuación, se habló sobre la intervención en crisis (Muñoz-Fernández et al., 2020) que sirve para identificar o evaluar una o más soluciones inmediatas o a futuro que beneficien la toma de decisión. Se realizó una actividad grupal donde se plantearon diferentes problemáticas y los participantes buscaron soluciones para debatirlas. Al final se pidió una tarea donde debían seguir los pasos para resolver un problema previo. 
Sesión 5. Diseñada para compartir e intercambiar todo lo aprendido. Se comenzó compartiendo las excepciones que encontraron durante la semana y como se sintieron. Posteriormente, los participantes hicieron una carta de felicitación escrita desde un futuro cercano donde la persona se felicitaría a sí misma por haber sido capaz de seguir haciendo las cosas que sabe que le ayudan a mantenerse sin estrés y describirlas detalladamente; para que en un futuro, la persona pueda leerla y poner en práctica todo lo aprendido y trabajado en el taller. Para concluir el taller, los participantes compartieron su experiencia, se pidió que comentaran si notaron cambios o mejoras, así como sugerencias para próximos talleres. Al finalizar el taller, se enviaron las evaluaciones por correo electrónico para que los participantes las contestaran y las enviaran de regreso con la finalidad de evaluar si los niveles de estrés y miedo disminuyeron, y así poder determinar si el taller fue eficaz.

\section{Análisis estadístico}

Los análisis estadísticos se realizaron con el IBM SPSS 24.0, se estimaron los estadísticos descriptivos y considerando el tamaño de muestra se usó la prueba no paramétrica de rangos de Wilcoxon para comparar los puntajes antes y después del taller. Se presentan también los resultados de significancia estadística unilateral; además del tamaño del efecto estimado con la $d$ de Cohen.

\section{Consideraciones éticas}

La participación en el presente estudio fue por voluntad propia y la información que pudiera identificar a los participantes se trató de forma confidencial, además, se les notificó a los participantes que responder los cuestionarios se consideraba como su consentimiento para participar en el estudio y que tenían la libertad de abandonar las sesiones cuando quisieran. Asimismo, el proyecto de investigación fue evaluado y aprobado en sus aspectos éticos y metodológicos por investigadores del Cuerpo Académico en Psicología Social y de la Salud de la Universidad Autónoma de Nuevo León. 


\section{Resultados}

A continuación, se presentan los resultados obtenidos en los instrumentos de evaluación que se aplicaron:

En los resultados de la escala de miedo al COVID-19, se encontraron resultados variados, ya que siete de los participantes presentaron una disminución de miedo y tres participantes presentaron un aumento del miedo al COVID-19 al término del taller. La disminución del miedo varió desde 1 punto hasta 17 puntos de diferencia. Inicialmente, los participantes presentaban una media de $20.0(D E=6.5)$ y una mediana de 21.0. Al finalizar el taller presentaron una media de $14.7(D E=4.4)$ y una mediana de $13.5(Z=-1.836 ; p=$ .066 ; significancia unilateral $p=.033$ ), el tamaño de efecto fue $d=1.1$ (tabla 1).

\section{Tabla 1}

Comparación de puntuaciones antes y después de la intervención

\begin{tabular}{|c|c|c|c|c|c|c|c|c|}
\hline \multirow[b]{2}{*}{ Variable } & \multicolumn{3}{|c|}{$\begin{array}{l}\text { Antes de la } \\
\text { intervención }\end{array}$} & \multicolumn{3}{|c|}{$\begin{array}{l}\text { Después de la } \\
\text { intervención }\end{array}$} & \multirow{2}{*}{$\begin{array}{l}\text { Prueba de } \\
\text { rangos de } \\
\text { Wilcoxon* }\end{array}$} & \multirow{2}{*}{$\begin{array}{l}\text { Tamaño } \\
\text { del efecto }\end{array}$} \\
\hline & Mediana & Media & D.E. & Mediana & Media & D.E. & & \\
\hline $\begin{array}{l}\text { Miedo al COVID- } \\
19\end{array}$ & 21.0 & 20.0 & 6.5 & 13.5 & 14.7 & 4.4 & $\begin{array}{c}Z=-1.836 \\
p=.033\end{array}$ & $\mathrm{~d}=1.1$ \\
\hline Estrés percibido & 19.5 & 20.6 & 7.1 & 13.5 & 14.3 & 4.5 & $\begin{array}{c}\mathrm{Z}=-2.041 \\
\mathrm{p}=.020\end{array}$ & $d=1.06$ \\
\hline $\begin{array}{l}\text { Síntomas de estrés } \\
\text { por el COVID-19 }\end{array}$ & 32.0 & 35.6 & 10.1 & 30.0 & 28.2 & 6.5 & $\begin{array}{c}Z=-1.675 \\
p=.047\end{array}$ & $\mathrm{~d}=0.87$ \\
\hline $\begin{array}{l}\text { Estrés } \\
\text { postraumático }\end{array}$ & 15.5 & 18.6 & 10.4 & 15.0 & 16.6 & 5.3 & $\begin{array}{c}Z=-0.296 \\
p=.383\end{array}$ & $\mathrm{~d}=0.24$ \\
\hline $\begin{array}{l}\text { Interferencia por el } \\
\text { coronavirus }\end{array}$ & 33.0 & 31.4 & 8.6 & 25.0 & 24.4 & 7.6 & $\begin{array}{c}\mathrm{Z}=-1.887 \\
\mathrm{p}=.029\end{array}$ & $\mathrm{~d}=.086$ \\
\hline $\begin{array}{l}\text { Experiencias } \\
\text { positivas }\end{array}$ & 54.5 & 50.9 & 9.3 & 54.5 & 54.1 & 3.3 & $\begin{array}{c}Z=-0.821 \\
p=.205\end{array}$ & $d=-0.46$ \\
\hline
\end{tabular}

Respecto a la escala de estrés percibido relacionado con la pandemia de COVID-19, también se encontraron resultados variados, ya que siete de los sujetos presentaron disminución de estrés y tres de ellos 
aumentaron el estrés percibido al terminar el taller. La disminución del estrés percibido varió desde los 2 puntos hasta 20 puntos de diferencia. Inicialmente, los participantes presentaban una media de $20.6(D E=$ 7.1) y una mediana de 19.5. Al finalizar el taller presentaron una media de $14.3(D E=4.5)$ y una mediana de $13.5(Z=-2.041 ; p=.041 ;$ significancia unilateral $p=.020)$, el tamaño del efecto para esta diferencia fue de $d=1.06$.

En cuanto a los resultados del cuestionario de impacto psicológico del coronavirus, se obtuvieron diferentes resultados para las escalas que lo conforman. Respecto a las preguntas de inicio del cuestionario, siete participantes actualmente viven con personas que padecen alguna enfermedad crónica grave, un participante vive con un niño menor de 12 años y un participante vive con alguien dependiente que requiere mucha atención.

Respecto a las experiencias por el COVID-19, la mayoría antes y después del taller no han tenido síntomas similares a los del COVID-19 y ninguno ha sido contagiado ni hospitalizado por esto. Los contagios de amigos íntimos o familiares cercanos, pero que no viven con ellos, aumentaron de seis a ocho participantes. La muerte de un amigo o compañero de trabajo aumentó de uno a tres participantes. Tanto antes como después del taller mencionaron que si podían evitar el contagio tomando las medidas de prevención y que no creen que la situación del COVID-19 se vaya a solucionar a corto plazo.

En relación con las conductas asociadas al confinamiento, no se observó una gran diferencia entre las respuestas antes y después del taller. Sin embargo, se encontró que antes del taller solo tres de los diez participantes no salían habitualmente a trabajar, y al finalizar, la mitad de los participantes si salían y la otra mitad no. También, se vio que antes del taller todos los participantes consideraban que veían más la televisión a partir de estar en cuarentena, y después del taller, solo siete veían más la televisión. La mayoría, tanto antes como después, consideran que ven más películas, usan más el internet y hacen actividades que antes del confinamiento no tenían tiempo de hacer. 
Por otro lado, en los resultados de las conductas preventivas que llevan a cabo los participantes durante la pandemia, no hubo gran cambio en sus conductas. Se observó que, al salir de sus casas, todos siguen usando la mascarilla; sin embargo, antes nadie usaba guantes y ahora una persona comenzó a usarlos. También, antes un participante no mantenía la sana distancia y al finalizar el taller sí. Se notó un cambio al desinfectarse excesivamente o con frecuencia, anteriormente solo la mitad lo hacían, y al finalizar el taller, nueve de diez participantes lo llevaban a cabo.

En la escala de distrés, se pudo observar que en seis participantes se logró disminuir la sintomatología del estrés causada por la pandemia por COVID-19. Por otro lado, en tres participantes hubo un aumento de la sintomatología del estrés al término del taller. Y en un solo participante se mantuvo igual el puntaje, antes y después del taller. Inicialmente los participantes presentaban una media de $35.6(D E=10.1)$ y una mediana de 32.0. Al finalizar el taller presentaron una media de $28.2(D E=6.5)$ y una mediana de 30.0 en la escala de distrés $(Z=-1.675 ; p=.094$; significancia unilateral $p=.047)$, con un tamaño del efecto $d=0.87$.

En la escala de síntomas de estrés postraumático, se observó una variación en los resultados. Cuatro de los participantes mostraron una disminución en los resultados obtenidos por la escala, bajando desde 2 puntos hasta 20 puntos de diferencia. Solamente un participante se mantuvo con el mismo resultado antes y después del taller. Cinco de los participantes presentaron un aumento desde 1 punto, mientras que uno aumentó un total de 9 puntos en sus resultados. Inicialmente, los participantes presentaban una media de $18.6(D E=10.4)$ y una mediana de 15.5. Al finalizar el taller, presentaron una media de $16.6(D E=5.3)$ y una mediana de $15.0(Z=-0.296 ; p=.767$; significancia unilateral $p=.383)$, el tamaño del efecto para esta diferencia fue $d=0.24$.

En la escala de interferencia por el coronavirus, siete participantes mostraron que después del taller las cosas que interfieren en sus vidas, debido a la pandemia por COVID-19, disminuyeron notoriamente; bajando desde 2 puntos hasta 21 puntos de diferencia. Tres de los participantes presentaron un aumento en 
la escala de interferencia por COVID-19. Inicialmente, los participantes presentaban una media de 31.4 $(D E=8.6)$ y una mediana de 33.0. Al finalizar el taller presentaron una media de $24.4(D E=7.6)$ y una mediana de $25.0(Z=-1.887 ; p=.059$; significancia unilateral $p=.029)$ con un tamaño del efecto $d=.086$.

En la escala de experiencias positivas ante las adversidades, se encontró una variación de los resultados. Por una parte, en cinco de los participantes después del taller hubo un incremento de experiencias positivas ante las adversidades durante la pandemia por COVID-19. Subiendo desde dos puntos hasta 20 puntos. Por otro lado, cinco de los participantes presentaron una disminución de las experiencias positivas ante las adversidades, desde 2 puntos hasta 7. Inicialmente, los participantes presentaban una media de 50.9 $(D E=9.3)$ y una mediana de 54.5. Al finalizar el taller, presentaron una media de $54.1(D E=3.3)$ y una mediana de $54.5(Z=-0.821 ; \mathrm{p}=.411$; significancia unilateral $p=.205)$, este incremento presentó un tamaño del efecto $d=-0.46$.

Después de dos semanas de la última sesión, se realizó una sesión de seguimiento para discutir sobre cómo el taller ha influido en su vida diaria. Asistieron 8 de los 10 participantes, los que no pudieron asistir fue por cuestiones de tiempo. Se pudo percibir que los participantes se encontraban más resilientes ante la situación del COVID-19. Esto se pudo observar por comentarios como: “a pesar de que la situación ha ido en aumento he podido tomarme la situación de una manera diferente", "me he sentido más tranquila y no tan estresada como antes gracias a que estoy aplicando algunas técnicas que vimos en el taller ", "la actividad que se trata de darme cuenta lo que pienso, siento y hago, me ha ayudado a identificar cuando estoy mal y a que se debe", he aprendido a sobrellevar y controlar la ansiedad con la que llegué al taller" y "mi sueño ha mejorado". 


\section{Discusión}

Considerando los resultados presentados, puede observarse que al principio del taller los participantes presentaban un nivel medio de miedo y estrés hacia el COVID-19, y al finalizarlo, el grupo en promedio presentó niveles bajos de ambos. Los valores de significancia estadística unilateral y los tamaños del efecto estimados respaldan los resultados satisfactorios (tabla 1).

De acuerdo a la teoría transaccional del estrés (Lazarus y Folkman, 1984), el estrés se presenta ante la valoración que el individuo hace de una situación y de sus recursos para hacerle frente, al modificar la percepción de la situación y mejorar las habilidades de las personas para hacer frente a estas situaciones estresantes, no solo disminuye el estrés percibido, sino también las respuestas emocionales negativas producto del proceso de estrés. En este caso, puede observarse que el miedo disminuyó.

Durante el transcurso del taller, los participantes se mostraron participativos e involucrados tanto dentro como fuera de las sesiones ya que implementaban las técnicas aprendidas en su vida diaria. La empatía y escucha activa que se tenían entre los participantes logró crear un ambiente de confianza para lograr un mejor entendimiento de su situación ante el COVID-19. Asimismo, ayudó a que los participantes adquirieran diferentes herramientas de manejo y afrontamiento de las experiencias compartidas por sus compañeros.

Algunas dificultades que ocurrieron durante el proceso fueron debidas a que, en las últimas 3 sesiones, hubo un repunte de contagios en México. Esto afectó de manera negativa a los participantes ya que se notaban un poco más enfocados en lo negativo al buscar excepciones y al llevar a cabo las técnicas. Una de las posibles razones por la que dos de los participantes, que eran familiares, tuvieran un aumento en las evaluaciones post-tratamiento pudo deberse a que, dentro de su familia, el hermano de una e hijo de la otra participante sospechaba de posible contagio de COVID-19. Así mismo, un compañero de trabajo de una de ellas había fallecido durante el transcurso del taller, lo que aumentó su miedo hacia el COVID-19. 
Muñoz-Fernández et al. (2020) propusieron una intervención en crisis para adquirir estrategias de afrontamiento ante la crisis por COVID-19. Con la finalidad de brindar el apoyo necesario a los pacientes y tener una remisión de los síntomas, evitando de esta forma que se necesite de un tratamiento farmacológico. Al aplicar la técnica de intervención en crisis, se pudo observar cómo, poco a poco, resultó más sencillo para los participantes el poder encontrar soluciones que estaban dentro de su alcance para la crisis presentada. Muchas de estas soluciones se relacionaban con crisis presentadas en temas laborales o familiares. Este enfoque en búsqueda de soluciones fue retomado en la presente investigación.

Como se mencionó anteriormente, las técnicas de externalización y relajación de Jacobson han sido útiles para la adquisición de habilidades de afrontamiento ante el estrés académico (López Novelo y González Ramírez, 2017). Al aplicarlas en el taller realizado, se pudo observar que sí resultaron ser técnicas efectivas para los participantes. Estos fueron capaces de externalizar el miedo y el estrés de ellos, llegando a sentirse más tranquilos; y al combinarla con la relajación progresiva, esta tranquilidad y relajación fueron mayores al terminar la sesión.

La psicoeducación de estrés, miedo y su relación con el COVID-19 ayudó para que los participantes pudieran entender desde una perspectiva más amplia la situación. Asimismo, con la psicoeducación de ideas irracionales, pudieron comprender y analizar de una mejor manera sus emociones y cómo estaban relacionadas con las ideas distorsionadas ayudándolos a ser un poco más objetivos y realistas ante la situación actual. La psicoeducación es ampliamente utilizada en talleres grupales, un ejemplo es el estudio de Villarroel y González Ramírez (2015) para la reducción del estrés académico en estudiantes universitarios.

El taller se organizó de acuerdo a las fases de la inoculación al estrés; Ayala (2017) encontró que el uso de esta estrategia resultó ser positiva para la reducción de estrés en músicos universitarios. Al llevar a cabo el taller, se pudo observar que la aplicación de las fases durante las sesiones dio un resultado positivo, 
permitiendo una reducción del estrés presentado, relacionado con la pandemia en la mayoría de los participantes. Este cambio se puede observar en la evaluación del pre y post de la escala de estrés percibido relacionado con la pandemia de COVID-19.

Núñez Camarena (2019) utilizó diferentes estrategias para disminuir los niveles de estrés en padres con hijos con enfermedades crónicas. Estas estrategias fueron de comunicación, de liberar emociones y de manejar el rol del cuidador, así como técnicas de respiración. Estas técnicas resultaron ser bastante efectivas ya que se encontró una disminución significativa en el pre-test y post-test del estrés, así como una mejoría en su manera de comunicar y manejar sus emociones. La técnica de respiración pudo haber sido un buen complemento a nuestra investigación para que, en el momento que se presente el estrés, los participantes puedan usar esta herramienta para sobrellevar la situación.

En un programa cognitivo conductual sobre manejo del estrés en pacientes con enfermedad crónica (Alonso Gascón, 2017), se incluyeron técnicas similares a las seleccionadas para el presente estudio, como la psicoeducación, manejo de pensamientos irracionales, entre otras. Sin embargo, incluyeron mindfulness para manejar el estrés. Asimismo, en la última sesión, implementaron la prevención de recaídas para anticipar y afrontar los problemas que se aproximen en un futuro. Esto logró grandes resultados en la disminución tanto del estrés como de la ansiedad que presentaban los participantes. Por estos resultados, el uso de esta técnica pudo haber sido un buen complemento para el taller, ayudando a tener un mejor manejo del estrés a largo plazo y se recomienda su uso en futuras intervenciones.

\section{Conclusiones}

El objetivo de esta investigación se logró cumplir ya que la mayoría de los participantes reportaron una disminución tanto en el estrés percibido como en el miedo hacia el COVID-19. Estos resultados apoyan la evidencia de la efectividad de la integración de la terapia cognitivo-conductual y de la centrada en 
soluciones; en este caso, usando la inoculación al estrés y otras técnicas seleccionadas, aplicándolas en línea. Uno de los aspectos que favorecieron los resultados fue la escucha activa y respeto que se dio entre los participantes, así como su participación y compromiso.

Algunas limitaciones que se presentaron fueron que los participantes presentaban diferentes inconvenientes que les impedía tanto asistir al taller como llegar puntuales. Así mismo, la situación actual de la pandemia fue otro inconveniente ya que, como es una situación cambiante y nueva, las últimas tres semanas se presentó un repunte en el número de contagios en México. Esto provocó que los participantes experimentaran altos niveles de incertidumbre ante la situación, influyendo en el estrés y miedo que presentaban. Asimismo, el diseño del estudio presenta limitaciones al no contar con un grupo de control y el tamaño del grupo también es una limitación. Lo anterior implica que los resultados presentados no son generalizables.

Para futuras investigaciones se recomienda que el taller sea de más horas, pero con menos sesiones. Esta fue una recomendación de los participantes ya que mencionaban que no sentían que el tiempo asignado a cada sesión fuera suficiente. De igual forma, consideramos que esto sería de gran beneficio para que las sesiones no se vean limitadas por cuestiones externas.

\section{Conflicto de intereses}

Las autoras declaran no tener conflicto de intereses

\section{Financiamiento}

La presente investigación no contó con financiamiento. 


\section{Referencias}

Alonso Gascón, M. R. (2017). Evaluación de un programa cognitivo conductual sobre manejo del estrés en pacientes con enfermedad crónica [Tesis de grado, Universidad Miguel Hernández]. http://193.147.134.18/bitstream/11000/5961/1/ALONSO\%20Miguel\%20TFM.pdf

Andión, O. (2012). El papel de la ansiedad rasgo en el condicionamiento del miedo: una aproximación desde la metodología del reflejo de sobresalto potenciado por miedo [Tesis de doctorado, Universidad Autónoma de Barcelona]. https://www.tdx.cat/handle/10803/362639

Ayala, P. (2017). Inoculación al estrés y atención plena para la reducción del estrés en músicos universitarios. Estudio comparativo [Tesis de maestría, Universidad Autónoma de Nuevo León]. http://eprints.uanl.mx/16624/1/1080289836.pdf

Brooks, S. K., Webster, R. K., Smith, L. E., Woodland, L., Wessely, S., Greenberg, N. y Rubin, G. J. (2020). The Psychological Impact of Quarantine and how to Reduce it: Rapid Review of the Evidence. The Lancet, 395, 912 - 920. https://doi.org/10.1016/S0140-6736(20)30460-8

Caballo, V.E., Valenzuela, J., Anguiano, S., Irurtia, M., Salazar, I. \& López-Gollonet, C. (2006). La Evaluación del Estrés y los Trastornos de Adaptación. En V. Caballo (dir.). Manual para la evaluación clínica de los trastornos psicológicos. Trastornos de la edad adulta e informes psicológicos. Pirámide.

Campo-Arias, A., Pedrozo-Cortés, M. J. y Pedrozo-Pupo, J. C. (2020). Escala de estrés percibido relacionado con la pandemia de COVID-19: una exploración del desempeño psicométrico en línea. Revista Colombiana de Psiquiatría, 49 (4), 229-230. https://doi.org/10.1016/j.rcp.2020.05.005

Castellano-Tejedor, C., Torres-Serrano, M. y Cencerrado, A. (2021). Psychological Impact in the Time of COVID-19: A Cross-sectional Population Survey Study During Confinement. Journal of Health Psychology. Online first. https://doi.org/10.1177/1359105320985580 
Castellini, G., Rossi, E., Cassioli, E., Sanfilippo, G., Innocenti, M., Gironi, V., Silvestri, C., Voller, F. y Ricca, V. (2021). A Longitudinal Observation of General Psychopathology Before the COVID-19 Outbreak and During Lockdown in Italy. Journal of Psychosomatic Research, 141, 110328. https://doi.org/10.1016/j.jpsychores.2020.110328

González, M.T. y Juárez, L.L. (2016). Análisis funcional en terapia breve cognitivo conductual centrada en soluciones. Revista Electrónica de Psicología de Iztacala, 19 (3), 1027-1039. http://www.iztacala.unam.mx/carreras/psicologia/psiclin/vol19num3/Vol19No3Art10.pdf

Gordon, S. F. (2020). El COVID-19 y la salud mental: ¿cuáles son las consecuencias? Psicología Iberoamericana, 28(1). https://www.redalyc.org/articulo.oa?id=133963198003

Guo, Y. R., Cao, Q. D., Hong, Z. S., Tan, Y. Y., Chen, S. D., Jin, H. J., Tan, K. S., Wang, D. Y. y Yan, Y. (2020). The Origin, Transmission and Clinical Therapies on Coronavirus Disease 2019 (COVID-19) Outbreak- An Update on the Status. Military Medical Research, 7(11). https://doi.org/10.1186/s40779$\underline{020-00240-0}$

Jacobson, E. (1938). Progressive Relaxation: A Physiological and Clinical Investigation of Muscular States and Their Significance in Psychology and Medical Practice. University of Chicago Press.

Kwasi, D., Lin, C., Imani, V., Saffari, M., Griffiths, M.D. y Pakpour, A.H. (2020). The Fear of COVID-19 Scale: Development and Initial Validation. International Journal of Mental Health and Addiction, 19. https://doi.org/10.1007/s11469-020-00270-8

Lazarus, R. (2000). Estrés y emoción. Manejo e implicaciones en nuestra salud. Desclée de Brouwer.

Lazarus, R. y Folkman, S. (1984). Stress, Coping and Adaptation. Springer.

López Novelo, C. L. y González Ramírez, M. T. (2017). Programas para la adquisición de habilidades de afrontamiento ante el estrés académico para estudiantes universitarios de primero ingreso. Revista 
Electrónica de Psicología $\quad$ Iztacala, $\quad 20(4), \quad 1362-1385$. https://www.iztacala.unam.mx/carreras/psicologia/psiclin/vol20num4/Vol20No4Art7.pdf

Meichenbaum, D. (1987). Manual de inoculación al estrés. Ediciones Martínez Roca, S.A.

Montero, I. y León, O. (2005). Sistema de clasificación del método en los informes de investigación en Psicología. International Journal of Clinical and Health Psychology, 5(1), 115-127. https://www.redalyc.org/articulo.oa?id=33701007

Muñoz-Fernández S. I., Molina-Valdespino D., Ochoa-Palacios R., Sánchez-Guerrero O. y EsquivelAcevedo J. A. (2020). Estrés, respuestas emocionales, factores de riesgo, psicopatología y manejo del personal de salud durante la pandemia por COVID-19. Acta Pediátrica de México, 41 (1), S127-S136. https://www.medigraphic.com/pdfs/actpedmex/apm-2020/apms201q.pdf

Muñoz-Navarro, R., Malonda, E., Llorca-Mestre, A., Cano-Vindel, A. y Fernández-Berrocal, P. (2021). Worry about COVID-19 Contagion and General Anxiety: Moderation and Mediation Effects of Cognitive Emotion Regulation. Journal of Psychiatric Research, 137, 311-318. https://doi.org/10.1016/j.jpsychires.2021.03.004

Núñez Camarena, K. P. (2019). Programa de intervención para el manejo de estrés de factores del entorno familiar dirigido a padres con hijos con enfermedades oncológicas en Fanlyc [Tesis de licenciatura, Universidad especializada de las Américas]. http://repositorio2.udelas.ac.pa/bitstream/handle/123456789/270/KeniaNuñez.pdf? sequence=1\&isA1 lowed $=\mathrm{y}$

Ramírez Treviño, J. y Rodríguez Alcalá, J. C. (2012). El proceso en psicoterapia combinando dos modelos: centrado en soluciones y cognitivo conductual. Revista Electrónica de Psicología Iztacala, 15(2), 610635. https://www.iztacala.unam.mx/carreras/psicologia/psiclin/vol15num2/Vol15No2Art14.pdf

Salari, N., Hosseinian-Far, A., Jalali, R. Vaisi-Raygani, A., Rasoulpoor, S., Mohammadi, M., Rasoulpoor, S. y Khaledi-Paveh, B. (2020). Prevalence of Stress, Anxiety, Depression among the General 
Population during the COVID-19 Pandemic: A Systematic Review and Meta-analysis. Global Health, 16(57). https://doi.org/10.1186/s12992-020-00589-w

Sandín, B., Valiente, R. M., García-Escalera, J. y Chorot, P. (2020). Impacto psicológico de la pandemia de COVID-19: Efectos negativos y positivos en la población española asociados al periodo de confinamiento nacional. Revista de Psicopatología y Psicología Clínica, 25(1), 1-22. https://doi.org/10.5944/rppc.23676

Sierra, J. C., Ortega, V. y Zubeidat, I. (2003). Ansiedad, angustia y estrés: tres conceptos a diferenciar. Revista Mal-estar E Subjetividade, 3(1), 10-59. https://www.redalyc.org/articulo.oa?id=27130102

Turna, J., Zhang, J., Lamberti, N., Patterson, B., Simpson, W., Francisco, A. P., Goldman, C. y Van Ameringen, M. (2021). Anxiety, Depression and Stress during the COVID-19 Pandemic: Results from a Cross-sectional Survey. Journal of Psychiatric Research, 137, 96-103. https://doi.org/10.1016/j.jpsychires.2021.02.059

Urzúa, A., Vera-Villarroel, P., Caqueo-Urízar, A. y Polanco-Carrasco, R. (2020). La Psicología en la prevención y manejo del COVID-19. Aportes desde la evidencia inicial. Terapia Psicológica, 38(1), 103-118. http://dx.doi.org/10.4067/S0718-48082020000100103

Valero Cedeño, N. J., Vélez Cuenca, M. F., Duran Mojica, A. A. y Torres Portillo, M. (2020). Afrontamiento del COVID-19: estrés, miedo, ansiedad y depresión. Enfermería Investiga, Investigación, Vinculación, Docencia Gestión, 5(3), https://revistas.uta.edu.ec/erevista/index.php/enfi/article/view/913/858

Villarroel, A. L. y González Ramírez, M. T., (2015). Intervención cognitivo-conductual y centrada en soluciones para disminuir el estrés académico en estudiantes universitarios. Revista Electrónica de Psicología Iztacala, 18(4), 1363-1387. https://www.medigraphic.com/pdfs/epsicologia/epi2015/epi154d.pdf 
White, M. (1991). La externalización del problema y la reescritura de vidas y relaciones. Cuadernos de Terapia Familiar, 18, 31-59.

Zhang, J., Wu, W., Zhao, X. y Zhang, W. (2020). Recommended Psychological Crisis Intervention Response to the 2019 Novel Coronavirus Pneumonia Outbreak in China: A Model of West China Hospital. Precision Clinical Medicine, 3(1), 3-8. https://doi.org/10.1093/pcmedi/pbaa006

Cómo citar este artículo: Gómez Hoyt, B. M., Méndez Pruneda , K. P., Novak Villarreal , M. F. ., \& González Ramírez, M. T. (2021). Manejo del estrés y miedo al COVID-19 desde la integración de los enfoques cognitivo-conductual y centrado en soluciones. Psicumex, 11, 1-24, e408. https://doi.org/10.36793/psicumex.v11i2.408 